\title{
Evaluation of Dermatology Consultations in Internal Medicine Wards: An Analysis of 510 Cases from A Tertiary Center
}

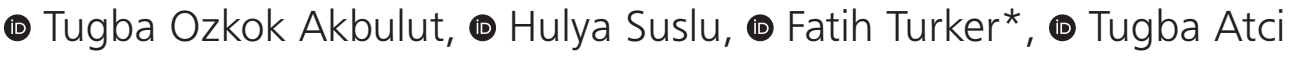 \\ University of Health Sciences Turkey, Haseki Training and Research Hospital, Clinic of Dermatology, Istanbul, Turkey \\ *University of Health Sciences Turkey, Haseki Training and Research Hospital, Clinic of Internal Medicine, Istanbul, Turkey
}

\section{Abstract}

\begin{abstract}
Aim: To evaluate the reasons for dermatology consultations in hospitalized patients in the internal medicine services and the treatments recommended based on these consultations.

Methods: The data of patients who were hospitalized in the internal medicine services between June 2018 and June 2020 were evaluated retrospectively including patients' demographics, reasons for the consultation, recommended additional tests and treatments by the dermatologist.

Results: A total of 510 patients (8.6\%) (51\% male, 49\% female, mean age $67.3 \pm 16.0$ years) requested dermatology consultations were reviewed among patients hospitalized in internal medicine services. The most common reason for the dermatology consultation was cutaneous and subcutaneous infections ( $n=156,30.6 \%)$ followed by decubitus ulcer $(n=94,18.4 \%)$ and vascular diseases ( $n=50$, $9.8 \%$ ). Topical and/or systemic treatments were recommended to $87.1 \%$ of the patients. Duration of hospitalization was longer in patients who requested dermatology consultations than patients not requested $(p<0.001)$.
\end{abstract}

Conclusion: Infectious causes and decubitus ulcer were the two most common reasons for dermatology consultations. Dermatology consultations are of great importance in hospitalized patients to decrease morbidity and the duration of hospitalization.

Keywords: Dermatology, consultation, internal medicine, decubitus ulcer

\section{Introduction}

Dermatology consultations requested from hospitalized patients, particularly in the internal medicine services, are of great importance for the diagnosis of the emerging skin manifestations, giving a clue about any accompanying systemic diseases and management of the disease (1). Dermatological problems are common in hospitalized patients and it is an important cause of morbidity (2).

There are various studies available in the literature evaluating the dermatology consultations (1-11). According to these studies, $21.5 \%$ to $45 \%$ of the dermatology consultations were requested from the internal medicine services, following emergency, pediatrics, surgery services. Additionally, cutaneous, subcutaneous infections, inflammatory skin diseases and drug eruptions were reported as the most common reasons for requesting a dermatology consultation in these studies. However, there are not many studies evaluating the reasons for requesting dermatology consultations focusing only on the hospitalized patients in internal medicine services (1). Determining the dermatological findings in hospitalized patients in internal medicine services may result with a decreased morbidity and a shorter duration of hospitalization.

Hence, the aim of this study is to retrospectively evaluate the dermatology consultations requested from patients hospitalized in the internal medicine services, and to scrutinize the reasons for requesting dermatology consultations, the diagnostic tests carried out and the recommended treatments for dermatological disorder.

Address for Correspondence: Tugba Ozkok Akbulut, University of Health Sciences Turkey, Haseki Training and Research Hospital, Clinic of Dermatology, Istanbul, Turkey Phone: +90 5542429026 E-mail: dderm08@hotmail.com ORCID: orcid.org/0000-0001-9995-2543 Received: 31.12.2020 Accepted: 27.02.2021 


\section{Methods}

\section{Study Design}

Dermatology consultations of the patients hospitalized in the internal medicine service of University of Health Sciences Turkey Haseki Training and Research Hospital between June 2018 and June 2020 were retrospectively analyzed based on their electronic database records. All these patients had been assessed by one of the dermatology specialists that work in our unit and who was on duty at the relevant date. The demographic characteristics of the patients, the reasons for the dermatology consultation requests, dermatological diagnoses, and the diagnostic tests recommended and implemented, and the recommended dermatological treatments were recorded. The ethics committee approval (number: 208/040620) of our study was obtained from the University of Health Sciences Turkey Haseki Training and Research Hospital Local Ethics Committee.

\section{Dermatological Assessment}

Dermatological diagnoses were categorized into eight main groups, namely, "cutaneous or subcutaneous infections", "inflammatory skin conditions", "drug eruptions", "autoimmune and bullous skin diseases", "skin manifestations of systemic diseases", "skin tumors", "vascular-related skin diseases" and "other" (if a diagnosis cannot be categorized under any of the foregoing groups). Additionally, other data such as the number of recurrent consultations and duration of the hospitalization regarding each patient were evaluated.

\section{Statistical Analysis}

The research data were worked up into Excel sheets. Statistical analyses were carried out using the Statistical Package for the Social Sciences Version 15.0 for Windows software. Descriptive statistics with regards to numerical variables were reported as mean, standard deviation, minimum and maximum values. The categorized groups were compared using the chi-square test. A statistical significance level of alpha was accepted as $p<0.05$. In the event that the comparison between the mean values of any two groups has resulted in a $\mathrm{p}$ (probability) value less than 0.05 indicating a statistically significant difference, the associated effect size was also calculated using the "Cohen's d" index in order to comprehend the level of any such statistically significant difference.

\section{Results}

One or more dermatology consultations were requested for the 510 (8.6\%) patients among 5957 patients hospitalized in the internal medicine services of our hospital between June 2018 and June 2020. The demographic characteristics of these patients are given in Table 1. The mean age of these patients was $67.3 \pm 16.0$ years. Of these patients, 260 (51\%) were male and $250(49 \%)$ were female. Cutaneous or subcutaneous infections were the most common reason $(n=156$, $30.6 \%)$ for requesting a dermatology consultation, with fungal infections (15.9\%) being the most common type of cutaneous infection registered within this group. The second most common reason for requesting a dermatology consultation was decubitus ulcer $(n=94,18.4 \%)$, followed by vascular diseases $(n=50,9.8 \%)$ and inflammatory skin diseases ( $n=45,8.8 \%$ ) (Table 2, Figure 1). Decubitus ulcers and vascular conditions were more common in patients $\geq 50$ years-old ( $p=0.008, p=0.021$, respectively), whereas autoimmune bullous diseases were more common in patients $<50$ years-old ( $p=0.023$, respectively) (Table 3 ).

Additional tests were required in $48.2 \%$ of these patients in order to confirm the dermatological diagnoses. The most common performed additional tests were as follows; direct fungus examination $(n=110)$, Wood's lamp examination $(n=49)$, skin punch biopsy $(n=30)$, bacterial culture $(n=28)$ and pathergy test $(n=25)$ (Figure 2$)$.

Topical treatments were recommended in 387 (87.1\%) of the patients for their dermatological disorders, either alone or in addition to any systemic therapy. Recommended topical treatments were as follows; topical antifungals (28.2\%), topical epithelizers (26.1\%), topical antibiotics (20.2\%), topical steroids (19.4\%), and wound dressings (15.9\%). A systemic treatment was recommended in $110(21.6 \%)$ patients, whereas no additional treatment was recommended in $66(12.9 \%)$ patients. Additionally, in $70(13.7 \%)$ patients, the respective findings revealed the need for seeking consultation from other specialties.

\begin{tabular}{|c|c|c|}
\hline & & n \\
\hline \multicolumn{2}{|c|}{$\begin{array}{l}\text { Patients for whom a dermatology } \\
\text { consultation was requested }\end{array}$} & $510 / 5.957(8.6 \%)$ \\
\hline \multirow[t]{2}{*}{ Gender } & Female & $250(49 \%)$ \\
\hline & Male & $260(51 \%)$ \\
\hline \multicolumn{2}{|c|}{ Age mean $\pm \mathrm{SD}$ (minimum-maximum) } & $67.3 \pm 16.0(20-100)$ \\
\hline \multirow{8}{*}{ Distribution by age groups } & $20-29$ & $12(2.4 \%)$ \\
\hline & 30-39 & $18(3.5 \%)$ \\
\hline & $40-49$ & $47(9.2 \%)$ \\
\hline & $50-59$ & $79(15.5 \%)$ \\
\hline & $60-69$ & $105(20.6 \%)$ \\
\hline & $70-79$ & $115(22.5 \%)$ \\
\hline & $80-89$ & $115(22.5 \%)$ \\
\hline & $90-100$ & $19(3.7 \%)$ \\
\hline \multicolumn{3}{|l|}{ SD: Standard deviation } \\
\hline
\end{tabular}




\begin{tabular}{|c|c|c|}
\hline & $n$ & $\%$ \\
\hline Cutaneous/subcutaneous infections & 156 & 30.6 \\
\hline Dermatophytosis & 30 & 5.9 \\
\hline Candidiasis & 29 & 5.7 \\
\hline Cellulitis & 23 & 4.5 \\
\hline Onychomycosis & 13 & 2.5 \\
\hline Secondary infection & 13 & 2.5 \\
\hline Herpes zoster & 8 & 1.6 \\
\hline Herpes simplex & 6 & 1.2 \\
\hline Intertrigo & 6 & 1.2 \\
\hline Folliculitis/furuncles & 5 & 1.0 \\
\hline Scabies & 5 & 1.0 \\
\hline Pyoderma & 4 & 0.8 \\
\hline Pityriasis versicolor & 3 & 0.6 \\
\hline Impetigo & 3 & 0.6 \\
\hline Lymphangitis & 2 & 0.4 \\
\hline Pediculosis & 1 & 0.2 \\
\hline Viral exanthema & 1 & 0.2 \\
\hline Ecthyma & 1 & 0.2 \\
\hline Orf & 1 & 0.2 \\
\hline Abcess & 1 & 0.2 \\
\hline Erysipelas & 1 & 0.2 \\
\hline Drug eruptions & 27 & 5.3 \\
\hline $\begin{array}{l}\text { Drug eruption (maculopapular, acneiform, lichenoid } \\
\text { drug eruption) }\end{array}$ & 19 & 3.7 \\
\hline Drug induced ulcer & 5 & 1.0 \\
\hline Stevens-Johnson syndrome & 3 & 0.6 \\
\hline Inflammatory skin diseases & 45 & 8.8 \\
\hline Contact dermatitis & 9 & 1.8 \\
\hline Psoriasis & 6 & 1.2 \\
\hline Erythema nodosum & 6 & 1.2 \\
\hline Piyoderma gangrenozum & 4 & 0.8 \\
\hline Nummuler dermatitis & 4 & 0.8 \\
\hline Diaper dermatitis & 4 & 0.8 \\
\hline Urticaria & 3 & 0.6 \\
\hline Seborrheic dermatitis & 3 & 0.6 \\
\hline Rosacea & 2 & 0.4 \\
\hline Lichen planus & 1 & 0.2 \\
\hline Angioedema & 1 & 0.2 \\
\hline Ichthyosis & 1 & 0.2 \\
\hline Hidradenitis suppurativa & 1 & 0.2 \\
\hline Vascular pathologies & 50 & 9.8 \\
\hline Stasis dermatitis & 28 & 5.5 \\
\hline Vasculitis & 7 & 1.4 \\
\hline Ecchymosis & 6 & 1.2 \\
\hline Acrocyanosis & 2 & 0.4 \\
\hline
\end{tabular}

\begin{tabular}{|l|l|l|}
\hline $\begin{array}{l}\text { Vascular malformation (hemangioma, venous } \\
\text { lacquer) }\end{array}$ & 2 & 0.4 \\
\hline Petechiae-purpura & 2 & 0.4 \\
\hline Thrombophlebitis & 2 & 0.4 \\
\hline Chronic lymphedema & 1 & 0.2 \\
\hline Autoimmune and bullous diseases & $\mathbf{1 6}$ & $\mathbf{3 . 1}$ \\
\hline Bullous pemphigoid & 7 & 1.3 \\
\hline Linear IgA bullous dermatosis & 1 & 0.2 \\
\hline Bullous systemic lupus erythematosus & 1 & 0.2 \\
\hline Acquired epidermolysis bullosa & 1 & 0.2 \\
\hline Skin tumors & $\mathbf{1 6}$ & $\mathbf{3 . 1}$ \\
\hline Benign neoplasm of skin & 8 & 1.6 \\
\hline Cutaneous T-cell lymphoma & 4 & 0.8 \\
\hline Basal cell carcinoma & 2 & 0.4 \\
\hline Cutaneous metastasis & 1 & 0.2 \\
\hline Kaposi's sarcoma & 1 & 0.2 \\
\hline Skin manifestations of systemic diseases & $\mathbf{2 3}$ & $\mathbf{4 . 5}$ \\
\hline Diabetic foot & 16 & 3.1 \\
\hline Uremic pruritus & 4 & 0.8 \\
\hline Diabetic dermatopathy & 1 & 0.2 \\
\hline Eruptive xanthoma & 1 & 0.2 \\
\hline Sclerodactyly & 1 & 0.2 \\
\hline Other (unclassified) & $\mathbf{1 9 7}$ & $\mathbf{3 8 . 6}$ \\
\hline Decubitus ulcer & 94 & 18.4 \\
\hline Pruritus & 31 & 6.1 \\
\hline Callus & 10 & 5.1 \\
\hline Keloid & 6 & 1.2 \\
\hline Artificial dermatosis & 6 & 1.2 \\
\hline Prurigo nodularis & 3 & 0.6 \\
\hline Perforating dermatosis & 3 & 0.6 \\
\hline $\begin{array}{l}\text { Other (terra-firma forme dermatosis, postlesionel } \\
\text { pigmentation, chloasma etc.) }\end{array}$ & 44 & 8.6 \\
\hline
\end{tabular}

It was also recommended to evaluate some patients $(n=8$, $1.6 \%$ ) in terms of malignancies, which may associate with the dermatological disorder.

A single dermatology consultation was deemed to be sufficient in $87.3 \%$ of the patients, whereas in $65(12.7 \%)$ patients recurrent consultations were required. The reasons indicated for requesting a recurrent consultation were as follows; newly developed dermatological problems ( $n=30$, $5.9 \%$ ), unresponsiveness to the treatment recommended in the previous consultation $(n=22,4.3 \%)$, and follow-up of the dermatological condition ( $n=13,2.5 \%)$.

While the mean duration of hospitalization of patients, requested a dermatology consultation was $6.72 \pm 6.86$ days (median: 5 days, range: $1-48$ days); it was $4.27 \pm 4.85$ days (median: 3 days, range: 1-62 days) for whom no dermatology consultation was requested. There was a statistically significant difference between these two 


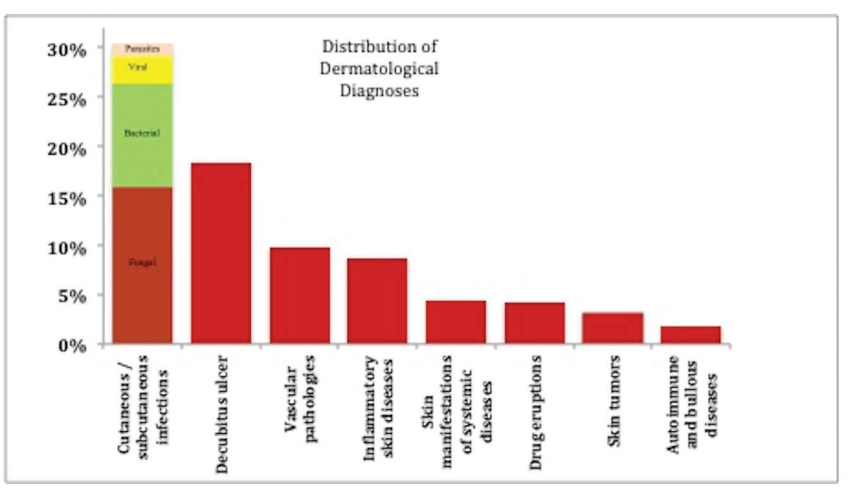

Figure 1. Distribution of dermatological diagnoses

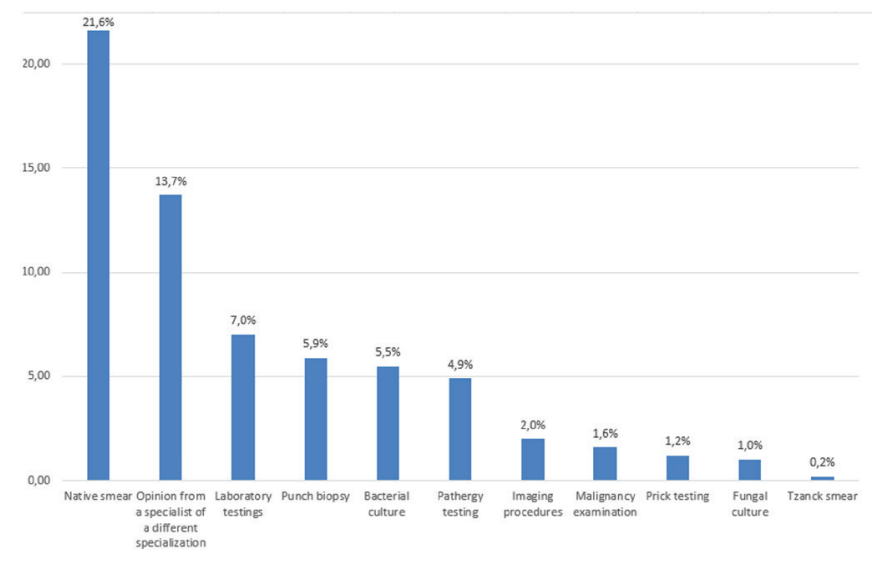

Figure 2. The proportion of each additional test and/or referrals based on the total of tests and/or referrals requested

\begin{tabular}{|c|c|c|c|c|c|}
\hline & \multicolumn{5}{|c|}{ Age } \\
\hline & \multicolumn{2}{|c|}{$<50$ years } & \multicolumn{2}{|c|}{$\geq 50$ years } & \multirow[b]{2}{*}{$p$} \\
\hline & $n$ & $\%$ & $\mathbf{n}$ & $\%$ & \\
\hline $\begin{array}{l}\text { Cutaneous/subcutaneous } \\
\text { infections }\end{array}$ & 22 & 28.6 & 134 & 30.9 & 0.677 \\
\hline Drug eruptions & 3 & 3.9 & 24 & 5.5 & 0.783 \\
\hline Inflammatory skin diseases & 9 & 9.1 & 36 & 7.4 & 0.605 \\
\hline Vascular pathologies & 2 & 2.6 & 48 & 11.1 & 0.021 \\
\hline $\begin{array}{l}\text { Autoimmune and bullous } \\
\text { diseases }\end{array}$ & 6 & 7.8 & 10 & 2.3 & 0.023 \\
\hline Skin tumors & 1 & 1.3 & 15 & 3.5 & 0.487 \\
\hline Decubitus ulcer & 7 & 9 & 87 & 20.1 & 0.008 \\
\hline $\begin{array}{l}\text { Skin manifestations of } \\
\text { systemic diseases }\end{array}$ & 4 & 5.2 & 19 & 4.4 & 0.765 \\
\hline
\end{tabular}

groups in terms of the mean duration of hospitalization $(p<0.001)$. Cohen's d effect size analysis value was calculated as 0.48 , revealing an effect size that is between medium effect (0.5) and small effect (0.2), and is thus considered significant.

\section{Discussion}

In this study, the dermatology consultations requested for patients hospitalized in the internal medicine service of a tertiary health center were reviewed in terms of the reasons for requesting a dermatology consultation and the treatments recommended based on these consultations.

Among the patients hospitalized in the internal medicine service, a dermatology consultation was requested for 510 patients (8.6\%). The most common reasons indicated for the dermatology consultations requested for these patients were cutaneous and subcutaneous infections, representing $30.6 \%(n=156)$ of them. Decubitus ulcer ( $n=94,18.4 \%$ ) was the second most common reason for requesting a dermatology consultation, which was followed by vascular pathologies $(n=50,9.8 \%)$ including stasis dermatitis as the most commonly observed type of vascular pathology, and inflammatory skin diseases $(n=45$, 8.8\%).

Similar to the results of our study, the most common reasons for requesting a dermatology consultation reported in the literature were infectious causes $(18.5 \%$ and $29.8 \%)$ and inflammatory diseases $(16.6 \%$ and $33.1 \%)(7,8)$. On the other hand, Lorente-Lavirgen et al. (10) reported inflammatory diseases (36.2\%) as the most common reason for requesting a dermatology consultation, followed by autoimmune diseases. This result may be due to the fact that the mean age of the patients included in the study of Lorente-Lavirgen et al. (10) was around 50 and that their hospital contains a globally accepted rheumatology unit.

In terms of etiological agents, it was observed that more than half of the infectious causes $(n=81)$ were skin fungal infections. Similar to the results of our study, Mancusi and Festa Neto (11) also reported that fungal infections accounted for nearly half of the infectious causes. On the other hand, there are also studies in which bacterial agents were reported as the most common infectious cause in the literature (4).

The fact that $85 \%$ of our patients were over the age of 50 and $70 \%$ of them were over the age of 65 which may be the reason for the relatively higher frequency of fungal infections found in our study. A relatively higher frequency of vascular skin pathologies (mainly stasis dermatitis), the third most common reason (9.8\%) for requesting a among dermatology consultations in our study, may also be attributed to the same reason. Similarly, Storan et al. (8) and Mancusi and Festa Neto (11) reported that stasis dermatitis and vascular pathologies were seen in $7.3 \%$ and $5 \%$ of the patients, respectively. 
Distribution of the dermatology consultations regarding age revealed that decubitus ulcers and vascular conditions were more common in patients $\geq 50$ years-old ( $p=0.008, p=0.021$, respectively), whereas autoimmune bullous diseases were more common in patients $<50$ years old ( $p=0.023$, respectively). These results are compatible with the results reported in comparable studies (12-14).

Additional medical workup including Tzanck smear, Wood's lamp examination, skin punch biopsy, ie; may be required at the diagnosis of the dermatological diseases in patients requested consultations. Additional medical workup was requested in $48.2 \%$ of the patients included in our study, and this ratio is compatible with similar studies ranging from $6.4 \%$ to $48 \%(3,5,11)$. However, certain tests specific to the field of dermatology such as the patch test, were not included in the requested medical workup, as it is recommended in our hospital to be performed under elective conditions following the discharge of the patient from the internal medicine service.

In our study, skin tumor diagnosis was made in 3.1\% of the patients for whom a dermatology consultation was requested. Among these patients, 4 patients had cutaneous T-cell lymphoma, 2 patients had basal cell carcinoma, 1 patient had cutaneous metastasis, and 1 patient had Kaposi's sarcoma. In comparison, Tay et al. (7) reported neoplasia in $4.8 \%$ of the patients, whereas Mancusi and Festa Neto (11) reported benign and malignant neoplasia in $6.7 \%$ of the patients.

Number of recurrent consultations requests has also been discussed in the literature as an indirect indicator of whether the dermatological problem is remedied or not. Fischer et al. (4) (reported that a single dermatology consultation was sufficient in $85 \%$ of the cases, compared to $71.8 \%$ and $60.9 \%$ of the cases in the studies of Penate et al. (5) and Connolly and Silverstein (3), respectively. Similarly, in our study, a single consultation was deemed to be sufficient in $87.3 \%$ of the cases. In terms of treatments recommended within the scope of dermatology consultations; Connolly and Silverstein (3) reported that topical treatments were recommended to $80.1 \%$ of the patients, and that systemic treatments were recommended to $19.1 \%$ of the patients (3). In comparison, in our study, topical treatments were recommended to $87.1 \%$ of the patients either alone or in addition to a systemic treatment. In the light of these data, it can be concluded that a single dermatology consultation is usually enough to resolve dermatological conditions and recurrent dermatology consultations are rarely needed, and topical treatments are often sufficient for these patients.

In our study, the duration of hospitalization was found to be significantly longer in patients, for whom a dermatology consultation was requested, compared to other patients. Similarly, it was stated in the study conducted by Philips et al. (9) that the median duration of hospitalization was 11 days in patients for whom a dermatology consultation was requested, compared to only 5 days in patients, for whom a dermatology consultation was not needed $(p=0.001)$. This result suggests that the rate of elective consultation requests, particularly for reasons of fungal infections, may increase as the duration of hospitalization get longer. Another reason for the significantly longer duration of hospitalization in patients for whom a dermatology consultation was requested may be that dermatological findings are cross-examined in patients with longer hospitalizations in order to have a clue in the underlying diseases, and symptoms such as decubitus ulcer and drug eruptions may emerge in patients with longer duration of hospitalization.

\section{Study Limitations}

An important limitation of this study is that it was based on hospital data system records and carried out retrospectively. In addition, there was not any data about the period between the time of emergence of the dermatological condition and the time of requesting the dermatology consultation, as well as about the correlation between the pre-diagnosis made by the internal medicine physician and the diagnosis made by a dermatologist. Another limitation of our study was not including the data of dermatology consultations requested from subspecialties of internal medicine of our hospital. In addition patients requested consultations were evaluated by various dermatologists which may result in to a challenge for diagnosis in this respect.

\section{Conclusion}

It was found that infectious causes and decubitus ulcers constitute a major part of the reasons for requesting dermatology consultations in patients hospitalized in the internal medicine service, and it has been established that dermatology consultations are of great importance in resolving problems associated with dermatological conditions diagnosed during hospitalization, such as prolonged hospitalization, additional economic costs and workforce loss. Therefore, it is recommended to provide trainings for the internal medicine and dermatology residents on the diagnosis and management of these diseases and for the nurses and auxiliary healthcare personnel working in the internal medicine service team on the preventive measures, especially with respect to decubitus ulcers.

\section{Authorship Contributions}

Concept: T.O.A., H.S., F.T., Design: T.O.A., T.A., Data Collection or Processing: H.S., F.T., Analysis or Interpretation: T.O.A., T.A., Literature Search: T.O.A., Writing: T.O.A. 
Conflict of Interest: No conflict of interest was declared by the authors.

Financial Disclosure: The authors declared that this study received no financial support.

\section{References}

1. Arakaki RY, Strazzula L, Woo E, Kroshinsky D. The impact of dermatology consultation on diagnostic accuracy and antibiotic use among patients with suspected cellulitis seen at outpatient internal medicine offices: a randomized clinical trial. JAMA Dermatol 2014;150:1056-61.

2. Galimberti F, Guren L, Fernandez AP, Sood A. Dermatology consultations significantly contribute quality to care of hospitalized patients: a prospective study of dermatology inpatient consults at a tertiary care center. Int J Dermatol 2016;55:547-51.

3. Connolly DM, Silverstein DI. Dermatology consultations in a tertiary care hospital: A retrospective study of 243 cases. Dermatol Online J 2015;21:13030.

4. Fischer M, Bergert $H$, Marsch WC. Das dermatologische Konsil [The dermatologic consultation]. Hautarzt 2004;55:543-8.

5. Peñate $Y$, Guillermo $N$, Melwani $P$, Martel R, Borrego L. Dermatologists in hospital wards: an 8-year study of dermatology consultations. Dermatology 2009;219:225-31.

6. Fernandes IC, Velho G, Selores M. Dermatology inpatient consultation in a Portuguese university hospital. Dermatol Online J 2012;18:16.
7. Tay LK, Lee HY, Thirumoorthy T, Pang SM. Dermatology referrals in an East Asian tertiary hospital: a need for inpatient medical dermatology. Clin Exp Dermatol 2011;36:129-34.

8. Storan ER, McEvoy MT, Wetter DA, et al. Experience of a year of adult hospital dermatology consultations. Int J Dermatol 2015;54:1150-6.

9. Phillips GS, Freites-Martinez A, Hsu M, et al. Inflammatory dermatoses, infections, and drug eruptions are the most common skin conditions in hospitalized cancer patients. J Am Acad Dermatol 2018;78:1102-9.

10. Lorente-Lavirgen Al, Bernabeu-Wittel J, Pulpillo-Ruiz Á, de la Torre-García JM, Conejo-Mir J. Inpatient dermatology consultation in a Spanish tertiary care hospital: a prospective cohort study. Actas Dermosifiliogr 2013;104:148-55.

11. Mancusi S, Festa Neto C. Inpatient dermatological consultations in a university hospital. Clinics (Sao Paulo) 2010;65:851-5.

12. Yalçin B, Tamer E, Toy GG, Oztaş $P$, Hayran M, Alli N. The prevalence of skin diseases in the elderly: analysis of 4099 geriatric patients. Int J Dermatol 2006;45:672-6.

13. Alpsoy E, Akman-Karakas A, Uzun S. Geographic variations in epidemiology of two autoimmune bullous diseases: pemphigus and bullous pemphigoid. Arch Dermatol Res 2015;307:291-8.

14. Mervis JS, Phillips TJ. Pressure ulcers: Pathophysiology, epidemiology, risk factors, and presentation. J Am Acad Dermatol 2019;81:881-90. 